\title{
Experimental Autoimmune Uveitis: An Intraocular Inflammatory Mouse Model
}

\author{
Chantelle E Bowers ${ }^{1}$, Virginia L Calder ${ }^{1}$, John Greenwood ${ }^{1}$, Malihe Eskandarpour ${ }^{1}$ \\ ${ }^{1}$ UCL Institute of Ophthalmology, University College London
}

\section{Corresponding Author}

Malihe Eskandarpour

m.eskandarpour@ucl.ac.uk

\section{Citation}

Bowers, C.E., Calder, V.L.,

Greenwood, J.,

Eskandarpour, M. Experimental

Autoimmune Uveitis: An Intraocular

Inflammatory Mouse Model. J. Vis.

Exp. (179), e61832, doi:10.3791/61832 (2022).

\section{Date Published}

January 12, 2022

\section{DOI}

$10.3791 / 61832$

URL

jove.com/video/61832

\section{Abstract}

Experimental Autoimmune Uveitis (EAU) is driven by immune cells responding to self-antigens. Many features of this non-infectious, intraocular inflammatory disease model recapitulate the clinical phenotype of posterior uveitis affecting humans. EAU has been used reliably to study the efficacy of novel inflammatory therapeutics, their mode of action and to further investigate the mechanisms that underpin disease progression of intraocular disorders. Here, we provide a detailed protocol on EAU induction in the C57BL/6J mouse - the most widely used model organism with susceptibility to this disease. Clinical assessment of disease severity and progression will be demonstrated using fundoscopy, histological examination and fluorescein angiography. The induction procedure involves subcutaneous injection of an emulsion containing a peptide (IRBP1-20) from the ocular protein interphotoreceptor retinoid binding protein (also known as retinol binding protein 3), Complete Freund's Adjuvant (CFA) and supplemented with killed Mycobacterium tuberculosis. Injection of this viscous emulsion on the back of the neck is followed by a single intraperitoneal injection of Bordetella pertussis toxin. At the onset of symptoms (day 12-14) and under general anesthesia, fundoscopic images are taken to assess disease progression through clinical examination. These data can be directly compared with those at later timepoints and peak disease (day 20-22) with differences analyzed. At the same time, this protocol allows the investigator to assess potential differences in vessel permeability and damage using fluorescein angiography. EAU can be induced in other mouse strains - both wildtype or genetically modified - and combined with novel therapies offering flexibility for studying drug efficacy and/or disease mechanisms.

\section{Introduction}

This protocol will demonstrate how to induce Experimental Autoimmune Uveitis (EAU) in the C57BL/6J mouse by a single subcutaneous injection of a retinal antigen in an emulsified adjuvant. Methods for monitoring and 
assessing disease progression will be detailed through fundoscopic imaging and histological examination, with measurement parameters outlined within. In addition, fluorescein angiography, a technique for examining retinal blood vessel structure and permeability will be discussed.

This EAU model recapitulates central features of noninfectious posterior uveitis in humans with regards to clinicopathologic characteristics and the basic cellular and molecular mechanisms that drive disease. EAU is mediated by Th1 and/or Th17 subsets of self-reactive $\mathrm{CD}^{+} \mathrm{T}$ lymphocytes, as shown in adoptive transfer experiments and with IFNy-depleted mice ${ }^{1}$. Much of our understanding of the potential roles for these cells in uveitis comes from studying EAU ${ }^{2}$ where both Th1 and Th17 cells are detected within the retinal tissues ${ }^{3}$. Often, EAU is used as a preclinical model to assess the utility of novel therapies in attenuating disease. Therapeutic approaches that have successfully modulated EAU disease have shown some efficacy in the clinic and reached FDA approved status. Examples of these are groups of immunoregulatory drugs such as the T cell-targeting therapies: cyclosporine, FK-506, and rapamycin ${ }^{4,5,6}$. Recently, interventions targeting novel pathways have also been explored in this model to investigate both mechanism and effect on disease outcome. These include targeting transcriptional regulation through chromatin reader Bromodomain Extra-Terminal (BET) proteins and $\mathrm{P}_{-}$ TEFb inhibitors ${ }^{3}$. Moreover, more conventional approaches such as a VLA-4 inhibitor have recently demonstrated suppression in EAU via modulation of effector $\mathrm{CD}^{+}{ }^{+}$Tells $^{7}$. In addition, targeting Th17 cells with TMP778, a RORyt inverse agonist, has also been found to significantly suppress $E A U^{8}$. Furthermore, this model offers an opportunity to study chronic autoimmune inflammation in the retina and the accompanying underlying mechanisms such as lymphocyte priming.

The primary readouts for EAU preclinical studies are clinical assessment by performing retinal fundoscopy imaging and less frequently, by assessing retinal integrity by Optical Coherence Tomography (OCT). Retinal histopathological evaluation and immunophenotyping of retinal cells by flow cytometry are then undertaken at termination. Fundoscopy is an easy-to-use live imaging system that allows for rapid and reproducible clinical assessment of the whole retina. For immunohistochemical assessments, the techniques are based on the preparation of retinal sections that allow us to study tissue architecture for the degree of inflammation and structural damage ${ }^{9}$. The assessment criteria and conventional scoring systems, for all techniques used, will be outlined within this protocol. The extent of damage recorded using fundoscopic imaging often closely correlates with histological changes. This dual approach to monitoring and assessing disease severity affords greater sensitivity and more reliable measurement outcomes.

EAU is a well-established, commonly used model for preclinical testing and investigation of immune-mediated eye disease. This model is reliable and reproducible with $>95 \%$ disease incidence and generates comprehensive data that can be used to validate or repudiate new therapies for the treatment of intraocular inflammatory disease that represents a major cause of working-age blindness worldwide ${ }^{10}$.

\section{Protocol}

All experiments were performed in accordance with the UK Animals (Scientific Procedures) Act of 1986, and institutional Animal Welfare and Ethical Review Body (AWERB) guidelines. 


\section{Housing C57BL/6J mice}

1. House mice in a specific pathogen free environment, on a 12 hour light-dark cycle and food and water available ad libitum.

2. Perform all experiments on adult female C57BL/6J (females are preferentially chosen as there is an incidence of women to men 1.4 to 1 in uveitis patients). Randomize female C57BL/6J mice between 6-8 weeks old by weight and age and housed in individual ventilated cages (IVC).

\section{Immunization of C57BL/6 mice}

1. IRBP1-20 - CFA Emulsion Preparation

NOTE: Emulsion preparation is essential to the reproducibility and incidence of disease; as such, every effort must be made to maintain consistency throughout the preparation process and across experiments. When preparing the emulsion, loss should be accounted for in the calculations of all reagents beforehand. This loss can be approximately $1.5 x$ (or $50 \%$ extra of the volume prepared), based on the number of mice planned for immunization. Please see the following example below. To immunize 10 mice, prepare 15 mice and use $400 \mu \mathrm{g}$ (peptide per $20 \mathrm{~g}$ mouse) $\times 15$ mice $=6 \mathrm{mg}$. Each mouse should receive $200 \mu \mathrm{L}$ for immunization (3 $\mathrm{mL}$ total). The final volume comprises a 1:1 ratio of peptide solution and CFA, hence $1.5 \mathrm{~mL}$ of peptide solution and $1.5 \mathrm{~mL}$ of CFA.

1. Prepare all sterile solutions in a laminar flow cabinet using aseptic techniques.

2. Weigh out desired amount ( $400 \mu \mathrm{g}$ per $20 \mathrm{~g}$ mouse) of human IRBP1-20 (LAQGAYRTAVDLESLASQLT) lyophilized peptide. Dissolve peptide in 100\% DMSO. Store stock in lyophilized form at $-20^{\circ} \mathrm{C}$.

NOTE: To ensure the powder is fully dissolved each flake must make contact with the DMSO first and show no sign of residual solid. Add PBS in small portions to reach the final volume. Do not mix with a vortex, instead use gentle agitation with a pipette. The final concentration of DMSO should not exceed $1 \%$ of the total peptide preparation volume. Preparing the emulsion in a $20 \mathrm{~mL}$ plastic tube with a tapered bottom should allow better accessibility of DMSO to the lyophilized powder.

3. Add DMSO-PBS peptide solution at $1: 1 \mathrm{v} / \mathrm{v}$ to CFA which has already been supplemented with $1.5 \mathrm{mg} /$ $\mathrm{mL}$ killed Mycobacterium tuberculosis, to give a final concentration of $2.5 \mathrm{mg} / \mathrm{mL}$. Add dropwise, pipetting gently and frequently to form a viscous and evenly distributed emulsion.

4. Aerate the peptide solution and CFA using a 1000 $\mu \mathrm{L}$ pipette (set to $700 \mu \mathrm{L}$ to prevent further loss) and pipette to generate a creamy thick consistency. This technique involves using the pipette to repeatedly aspirate up and down until reaching the desired thickness. For optimal results, ensure that the antigen solution and adjuvant are mixed thoroughly before injecting.

2. Intraperitoneal injection of pertussis toxin

1. Suspend $1.5 \mu \mathrm{g}$ Bordetella pertussis toxin in $100 \mu \mathrm{L}$ of RPMI 1640 media supplemented with $1 \%$ mouse serum $^{11}$.

2. Perform the i.p. injection with a sterile syringe and $23 G$ needle. 
NOTE: In order to avoid disturbances to the injection site, the pertussis toxin must be administered before injecting the antigen.

3. Temporarily transfer each mouse to a separate cage to receive a single $100 \mu \mathrm{L}$ i.p. injection of Bordetella pertussis toxin.

3. Subcutaneous injection of IRBP emulsion

1. Next, inject the IRBP emulsion subcutaneously. This process requires two animal handlers appropriately attired with protection according to health and safety regulations.

2. Have one trained person lightly restrain the mouse on top of the cage in a scruff-like position, with their stomach facing downwards whilst the other trained person pinches the skin to form a tent-like structure on the back of the neck where the needle can be inserted to slot between the finger and thumb.

CAUTION: There is a danger of needle stick injury.

3. Once the needle is positioned, inject $200 \mu \mathrm{L}$ of the IRBP emulsion. When removing the needle, rotate the needle head to close the skin before pulling out and apply pressure afterward to the injection site to prevent reflux of the emulsion.

CAUTION: The emulsion must not make contact with the mouse skin or fur as this may cause irritation and in more severe cases, a lesion to develop. If this occurs, the area must be wiped immediately and thoroughly using $70 \%$ ethanol then dried.

NOTE: If the draining lymph nodes are needed for examination at the end of the study, the injection site will be different. In this instance, inject 100 $\mu \mathrm{L}$ to both sides of the flank subcutaneously. This will generate a stronger response at the draining inguinal lymph nodes, which can be excised at the time of harvesting. However, if the intended outcome is solely to develop EAU, a single injection of 200 $\mu \mathrm{L}$ at the back of the neck is preferable to avoid discomfort from multiple injection sites.

\section{Clinical Evaluation - Mouse Fundus Examination}

NOTE: Clinical disease is to be scored using fundus examination, via bright-field live imaging using a fundoscope and Discover software used for visualization.

1. At disease onset (day 12-14), sedate mice under general anesthesia using a combination of both Ketamine (50 $\mathrm{mg} / \mathrm{mL}$ ) and Domitor (Medetomidine; $1 \mathrm{mg} / \mathrm{mL}$ ). Dilute 1-part Domitor; 1.5 parts Ketamine and 2.5 parts sterile injectable water, then inject $100 \mu \mathrm{L}$ per $30 \mathrm{~g}$ intraperitoneally. Use $1 \mathrm{~mL}$ sterile syringes and 23G needles for the above combination of anesthesia.

2. Following this, monitor the mouse to ensure that all reflexes are lost and that it is unresponsive to stimuli.

3. Immediately after receiving the i.p. injection and whilst the mouse is still held in a scruff, apply $1 \%$ tropicamide and $2.5 \%$ phenylephrine topically to each eye for pupil dilation. Aim to completely cover the cornea with both dilating solutions. It may take a few minutes before the pupil is fully dilated.

4. Afterward, generously apply to the eye viscotears ointment and maintain throughout the imaging process in order to keep the eye fully lubricated and hydrated.

5. In the meantime, open the software (e.g., Discover), and set the fundoscope (e.g., Micron) to capture images under brightfield. Allocate each individual mouse a folder 
and label images with $\mathrm{R}$ or $\mathrm{L}$ according to each eye photographed.

6. Mount the mouse on a purpose-built stage for live visualization and position the microscope for full access to the retina.

7. To get an accurate representation of the disease, take images of the entire retinal area, covering all corners of the periphery in addition to the optic disc. To achieve this, adjust the eyepiece throughout. It is crucial for the eye to remain fully lubricated at all times throughout the imaging process; ensure this by topping up the eye ointment at a constant rate.

8. Refer to section 4 (below) at this stage to perform fluorescein angiography.

9. Once all imaging is complete, dilute anesthetic reversal anti-sedation (5 $\mathrm{mg} / \mathrm{mL}$ Antisedan) in injectable water and administer at $0.1 \mathrm{mg} / \mathrm{kg}$ i.p. Return the mouse to a cage and place on a pre-heated mat with access to a wet-soaked diet until recovery. Complete recovery is characterized by whole body movement and walking around the cage with steady gait, typically taking a few hours.

10. At the designated experimental endpoint (e.g., day 21-23), repeat steps 4.1-4.5 and take photographs of the entire retinal area again, covering the optic disc and all corners of the periphery to capture an accurate representation of disease.

\section{Fluorescein angiography}

1. To measure vessel leakage in these animals, whilst under anesthesia, give each mouse an injection of $2 \%$ fluorescein subcutaneously at the back of the neck and position such that the retina is centralized in the middle of the live image.

2. Set the fundoscope to a blue light excitation filter at 465-490 $\mathrm{nm}$. The light captured from excited fluorescein is between $520-530 \mathrm{~nm}$.

3. After $1.5 \mathrm{~min}$ post fluorescein injection, take a photograph of each retina and repeat again at 7 minutes. NOTE: Timing is critical for these events, if unable to capture both then just image one eye.

\section{Clinical Disease Scoring}

1. Base the clinical assessment on the severity of the following criteria: optic disc inflammation, retinal vessel cuffing, retinal tissue infiltrate and structural damage.

2. Award each of these parameters a score on a scale from 0 to 5 and the collective total is representative of clinical disease for the whole eye, with a maximum score of 20 obtainable per eye. Table 1 can be used as a guide for scoring criteria.

\section{Histology and Histological Scoring}

1. After killing the mice enucleate the eyes by prizing the eyelids apart for easy access to the entire eye.

2. Next, place curved forceps behind the globe with the intention of grasping the orbital connective tissue and optic nerve. Take care to avoid squeezing the globe.

3. For fixation, place the eye in $4 \%$ glutaraldehyde for a minimum of 15 minutes to minimize retinal detachment, and then transfer to $10 \%$ formaldehyde for at least $24 \mathrm{~h}$. 1-2 $\mathrm{mL}$ of fixative would give enough volume to cover two eyes.

4. Perform embedding in paraffin, sectioning on a microtome, and staining according to standard protocols. 
3-4 $\mu \mathrm{m}$ section thickness is recommended for any type of staining.

5. Perform histological examination of eyes using standard protocols for Hematoxylin and Eosin (H\&E) staining.

6. Assign scores on a scale of $0-4$, according to the criteria for EAU scoring, based on the extent of the immune cell infiltration within the retina and choroid, the disruption of the retinal layers, the degree of granuloma formation and the extent of retinal detachment, indicating retinal damage, as previously described (Agarwal 2013) and summarized in Table $2^{11}$.

\section{Representative Results}

In this protocol, we describe a step-by-step method for inducing a model of experimental autoimmune uveitis (EAU) by immunizing mice with a uveitogenic retinal peptide derived from IRBP. The assessment of disease employing widely used and readily accessible approaches are covered although these are not exclusive and may be added to, or partially replaced, by other imaging techniques. The first signs of EAU in C57BL/6J mice can be detected two weeks post-immunization and peak disease reached within three weeks as illustrated in Figure 1. Fundoscopic changes are classified during disease progression as inflammatory changes, which include retinal tissue, vascular and optic disc inflammation, and retinal structural damage (Figure 2) in addition to histological changes based on infiltrating immune cells and structural damage. These clinical and histopathological changes can be detected for up to 85 days post immunization, and graded and scored for evaluation proposes to study disease progression. In order to avoid unintentional bias in the qualitative visual scoring, the images should be evaluated by more than one expert and scorers require to be blinded to the treatment groups.

We show here how the clinical and histological scoring systems (Table 1 and Table 2) guide scientists to quantify EAU severity, to validate the efficacy of treatments and to explore the mechanism of drug action. Vascular leakage is also a pathological feature of the model and in human uveitis. We are showing examples of vascular leakage of fluorescein (Figure 3) as another method for assessing disease in this model. 


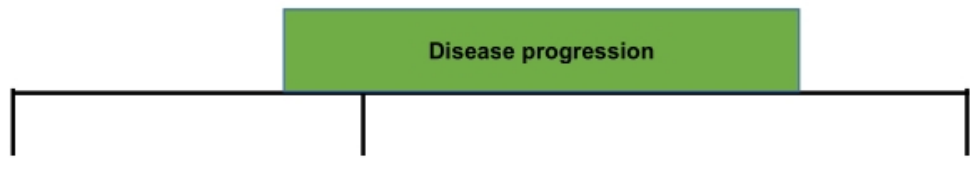

$12-14$

21-23

Figure 1. Schematic timeline of clinical and histological disease progression in IRBP1-20 induced EAU. A timeline marking the onset of infiltration and progression of IRBP1-20 induced EAU towards peak disease. From immunisation, the first signs of clinical disease, as detected by fundoscopic imaging and histopathological analysis, falls between days 12-14. The disease will then continue to progress, according to these parameters, until a peak is reached around day 21-23. Please click here to view a larger version of this figure. 


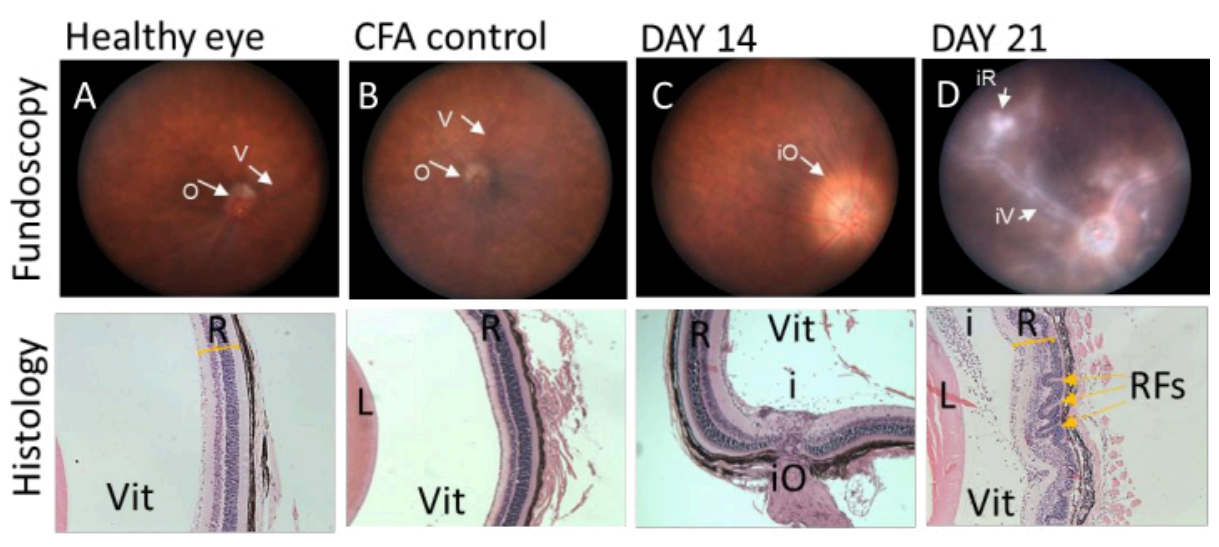

Figure 2. Representative fundoscopic images correlating with histological sections at different stages of IRBP1-20 induced EAU disease in C57BL/6J mice. Clinical fundoscopic and corresponding tissue images of C57BL/6J from the same animal immunised with IRBP1-20 peptide. (A and B) fundoscopic images and histological sections of eye obtained from healthy and CFA injected mice. Retina has no sign of inflammation and corresponding histology sections show preserved retinal layers. (C) Fundoscopic image of eye obtained from C57BL/6J mouse 14 days post immunization demonstrate classic signs of EAU, presenting with severe optic disc swelling in the early stage of disease, corresponding histology shows infiltrating immune cells into vitreous space. (D) Fundoscopic images of eye obtained from C57BL/6J mouse 21 days post immunization shows signs of vessel cuffing and infiltrating immune populations. Histology data demonstrates severe structural changes by retinal folding (yellow arrows). $V=$ vessel, $O=$ optic disk, $R=$ retina, $L=l e n s$, Vit=vitreous, $i O=$ inflamed optic disk, iV= inflamed vessel, iR= inflamed retina, $i=$ infiltrating cells in vitreous, $R F s=r e t i n a l$ folds. Please click here to view a larger version of this figure. 
A

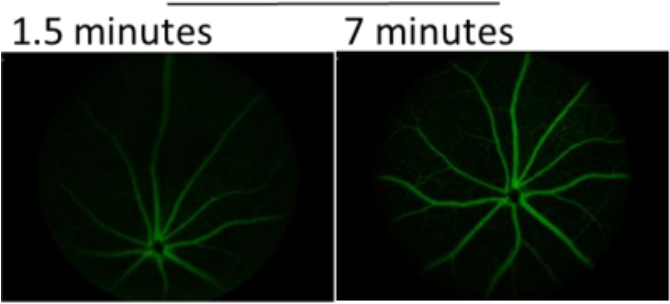

B

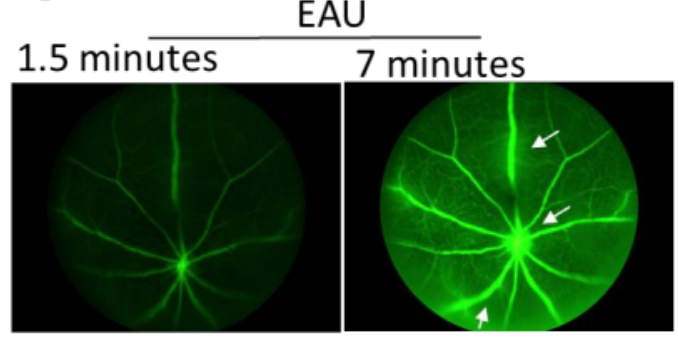

Figure 3. Representative images of fluorescent angiography taken using Micron III imaging system at peak disease.

C57BL/6J mice were injected subcutaneously with $2 \%$ fluorescein and images taken at various timepoints after circulation of the tracer. (A) CFA only control mouse taken at 1.5- and 7-minutes post fluorescein administration. (B) Representative images of IRBP1-20 immunized mice taken 1.5 and 7 minutes, respectively, after receiving fluorescein. White arrow indicates vessel leakage. Please click here to view a larger version of this figure. 


\begin{tabular}{|c|c|c|c|c|}
\hline Score & Optic Disc & Retinal Vessels & Retinal Tissue Infiltrate & Structural Damage \\
\hline 1 & Minimal Inflammation & 1-4 mild cuffings & $\begin{array}{l}\text { 1-4 small lesions } \\
\text { or } 1 \text { linear lesion }\end{array}$ & $\begin{array}{c}\text { Retinal lesions or } \\
\text { retinal atrophy involving } \\
1 / 4 \text { to } 3 / 4 \text { retina area }\end{array}$ \\
\hline 2 & Mild inflammation & $\begin{array}{l}>4 \text { mild cuffings or } \\
1-3 \text { moderate cuffing }\end{array}$ & $\begin{array}{l}\text { 5-10 small lesions } \\
\text { or 2-3 linear lesions }\end{array}$ & $\begin{array}{l}\text { Pan retinal atrophy } \\
\text { with multiple small } \\
\text { lesions (scars) or <3 } \\
\text { linear lesions (scars) }\end{array}$ \\
\hline 3 & Moderate inflammation & $>3$ moderate cuffings & $\begin{array}{l}>10 \text { small lesions } \\
\text { or }>3 \text { linear lesions }\end{array}$ & $\begin{array}{l}\text { Pan retinal atrophy with } \\
>3 \text { linear lesions or } \\
\text { confluent lesions (scars) }\end{array}$ \\
\hline 4 & Severe inflammation & $>1$ severe cuffing & Linear lesion confluent & $\begin{array}{l}\text { Retinal detachment } \\
\text { with folding }\end{array}$ \\
\hline 5 & $\begin{array}{l}{ }^{*} \text { Not visible (white out } \\
\text { or extreme detachment) }\end{array}$ & $\begin{array}{l}{ }^{*} \text { Not visible (white out } \\
\text { or extreme detachment) }\end{array}$ & $\begin{array}{l}{ }^{*} \text { Not visible (white out } \\
\text { or extreme detachment) }\end{array}$ & $\begin{array}{l}{ }^{*} \text { Not visible (white out } \\
\text { or extreme detachment) }\end{array}$ \\
\hline
\end{tabular}

Table 1. Conventional clinical scoring scale for evaluating EAU clinical disease severity. Table showing criteria used to evaluate the extent of disease severity in mice immunised with IRBP1-20. Scores were allocated according to the hallmarks outlined above being visible on the fundus images, each eye was given a total score out of twenty. ${ }^{*}$ Due to the obscuration of infiltrate and retinal detachment inside the posterior chamber cannot be assessed. Table adapted with permission from Xu H., et al., $2008^{8}$. 


\begin{tabular}{|c|c|}
\hline Grade & Criteria \\
\hline 0 & No change \\
\hline 0.5 (trace) & Mild inflammatory cell infiltration. No tissue damage \\
\hline 1 & $\begin{array}{l}\text { Infiltration; retinal folds and focal retinal detachments; } \\
\text { few small granulomas in choroid and retina, perivasculitis }\end{array}$ \\
\hline 2 & $\begin{array}{l}\text { Moderate infiltration; retinal folds, detachments and } \\
\text { focal photoreceptor cell damage; small to medium } \\
\text { sized granulomas, perivasculitis and vasculitis }\end{array}$ \\
\hline 3 & $\begin{array}{l}\text { Medium to heavy infiltration; extensive retinal folding with } \\
\text { detachments, moderate photoreceptor cell damage; medium } \\
\text { sized granulomatous lesions; subretinal neovascularization }\end{array}$ \\
\hline
\end{tabular}

Table 2. Histologically scoring EAU

Table showing criteria used to evaluate the severity of EAU based on histopathological features of disease. Scores were allocated according to the hallmarks outlined above on H\&E staining, each eye was given a total score out of four. Table adapted with permission from Agarwal et al. $2013^{11}$.

\section{Discussion}

Experimental animal models are necessary tools for studying disease pathogenesis and preclinical testing of novel therapeutic paradigms. In the current protocol, we have discussed a methodology for inducing, monitoring, and scoring EAU, an experimental model of intraocular inflammatory uveitis. This EAU model has more than 95\% disease incidence when all procedures are performed according to the protocol outlined herein, and results in the development of chronic, monophasic EAU. To achieve this incidence level, we stress the importance of antigen preparation and injection of the emulsion, both of which are detailed above. The main features of EAU in animals are retinal and/or choroidal inflammation, retinal vasculitis, photoreceptor destruction and loss of vision, all of which represent many essential clinicopathological features of human posterior uveitis ${ }^{12}$. Much of the understanding of the basic cellular and molecular mechanisms involved in uveitis derived from the induced EAU model as described herein. EAU can be induced in mice ${ }^{13}$ and rats $^{11}$ by active immunization with retinal antigens that are recognized by lymphocytes. These retinal antigens take many forms; IRBP (for mice) or retinal soluble antigen (S-Ag) for rats. Inducing EAU on a C57BL/6J background generates a more chronic form of the disease, with peak pathology observed three weeks post-immunization. By comparison, applying retinal antigen to a B10RIII background ${ }^{14}$ induces an acutemonophasic and clinically severe form of EAU where peak pathology typically presents within two weeks of induction, and disease subsides by week 3 .

Different IRBP epitopes have been tested in C57BL/6J mice and IRBP $1-20$ peptide has proven to be a reproducible 
model with a high incidence and severity levels. Recently a new uveitogenic epitope of IRBP, amino acid residues 651 to 670 of human IRBP have been reported to induce EAU with a higher clinical incidence and severe disease manifestation $^{11}$ and may be used in preference should this meet the scientific objectives. Since the impact of gut commensal microbiota and the activation of autoreactive $\mathrm{T}$ cell receptors (TCRs) is known to interfere with the onset of disease when applying different antigens ${ }^{15}$, we recommend for beginners in this field to use either hIRBP1-20 or hIRBP651-670 peptides at a dose titrated between 300-500 $\mu \mathrm{g}$ in order to achieve a reliable model. Indeed, variability in this model has been documented elsewhere with reports highlighting the importance of differences between housing systems and the microbiome which might impact disease severity and incidence ${ }^{15}$. Thus, more or less peptide antigen and pertussis toxin may be required.

There are a number of other models to which our described analysis can be performed. These include spontaneous uveitis that progresses in IRBP $\mathrm{T}$ cell receptor (TCR) transgenic $(\mathrm{R} 161 \mathrm{H})$ mice where ocular inflammation develops by $5-6$ weeks of age ${ }^{16}$. EAU can also be adoptively induced by transferring uveitogenic effector $\mathrm{CD} 4^{+} \mathrm{T}$ cells. Activated, IRBP-specific $\mathrm{CD}^{+} \mathrm{T}$ cells derived from primed mice can be used as a source of effector cells ${ }^{3,11}$. This model represents the effector phase of the disease while avoiding the complexities of using CFA in the inducible model.

Further to this, there are many advantages to using ocular inflammatory models as appropriate tools to investigate other inflammatory diseases, in particular, those with effector Th1 and Th17 subset pathology. The main advantages to using this model are the non-invasive and quantifiable methods for monitoring disease development and progression, which are fundoscopy and angiography. These non-invasive imaging systems allow easy access to neuronal tissues, that would otherwise be concealed behind protective anatomical barriers. Additional methods for monitoring disease progression include the application of OCT imaging, which is more sensitive than fundoscopic imaging in detecting cellular infiltrates, especially at the early stage of EAU onset. The technique allows multi-layer cross- and horizontal-sectional visualizations of the retina longitudinally and in a non-invasive fashion. In vivo OCT imaging adds information on retinal thickness that could not be obtained by fundoscopic and histological examinations ${ }^{17}$. Increasingly, the availability of even more sophisticated non-invasive imaging techniques, such as adaptive optics scanning laser ophthalmoscopy and multimodal imaging tools will further advance our capacity to investigate this disease in small rodents. Furthermore, the ability to dissect and isolate resident and infiltrating cell populations for deeper analysis of immunophenotypes, using techniques such as flow cytomety, affords great opportunities to deliver insightful information.

There are a few established scoring systems based on clinical criteria obtained from fundoscopy $8,9,18$. Whilst these differ slightly between ophthalmology research centers, all are reliable, correlate with histopathological features and are capable of accurately reflecting the severity of disease. In the current study, we refer to the scoring system developed by $\mathrm{Xu}$ et al. ${ }^{8}$. This system offers a more detailed assessment approach with greater numbers of clinical measurement parameters. It comprises a maximum score of 20 which introduces a wider window for scoring than alternative systems limited to a maximum of 5 . This is more important for further exploration within therapeutic approaches. Minimising operator error, however, is critical when using such a refined and detailed set of parameters and may require careful 
training of the operator and independent validation of the interpretation.

Here, we present a protocol to induce EAU in female C57BL/6 mice as there is an increased incidence of women : men 1.4:1 presenting with uveitis in the clinical setting. Nevertheless, the sex of the mice used for inducing autoimmune disease should be considered as this may affect the cytokine milieu ${ }^{11}$, and also reveal important differences in the way they respond to therapeutic intervention. Another consideration is the age of the mice at disease induction. For example, we have studied age-dependency of susceptibility in B10RIII mice and concluded that mice over 8 weeks of life have a lower incidence of EAU (unpublished study from our group).

In conclusion, animal models of intraocular disease have provided an invaluable tool to study human posterior uveitis and have facilitated development of novel therapies such as CsA. However, no animal model by itself reproduces the full spectrum of human uveitis, as each has unique characteristics that make it suitable for studying particular aspects of the disease. This EAU model is induced by autoimmunity through the application of IRBP peptide supplemented with adjuvants which trigger innate immune responses. However, it is not known if all forms of posterior uveitis in humans are autoimmune and if antigenic mimicry is a triggering factor. In addition, it is not clear whether there is an association with infection in triggering human uveitis. Nonetheless, the model described herein is a useful and reproducible generic model that can be used to glean useful information regarding aetiology, pathogenesis and therapy of this sight-threatening disease.

\section{Disclosures}

The authors have no conflicts of interest to declare with this work.

\section{Acknowledgments}

JG was awarded a UCL Impact Studentship and Rosetrees Trust funding to support CB. VC was in receipt of a research collaborative grant from Akari Therapeutics Inc. We would like to thank UCL Institute of Ophthalmology, Biological Service Unit especially Ms Alison O'Hara and her team for their technical support.

\section{References}

1. Lyu, C. et al. TMP778, a selective inhibitor of RORgammat, suppresses experimental autoimmune uveitis development, but affects both Th17 and Th1 cell populations. European Journal of Immunology. 48, 1810-1816 (2018).

2. Klaska, I. P., \& Forrester, J. V. Mouse models of autoimmune uveitis. Current Pharmaceutical Design. 21, 2453-2467 (2015)

3. Eskandarpour, M., Alexander, R., Adamson, P., \& Calder, V. L. Pharmacological Inhibition of Bromodomain Proteins Suppresses Retinal Inflammatory Disease and Downregulates Retinal Th17 Cells. Journal of Immunology. 198, 1093-1103 (2017).

4. Mochizuki, M. et al. Preclinical and clinical study of FK506 in uveitis. Current Eye Research. 11 Suppl, 87-95 (1992).

5. Nguyen, Q. D. et al. Intravitreal Sirolimus for the Treatment of Noninfectious Uveitis: Evolution through 
Preclinical and Clinical Studies. Ophthalmology. 125, 1984-1993 (2018).

6. Leal, I. et al. Anti-TNF Drugs for Chronic Uveitis in AdultsA Systematic Review and Meta-Analysis of Randomized Controlled Trials. Frontiers in Medicine (Lausanne). 6, 104 (2019).

7. Chen $\mathrm{YH}$ et al. Functionally distinct $\mathrm{IFN}-\mathrm{Y}^{+} \mathrm{IL}-17 \mathrm{~A}^{+}$ Th cells in experimental autoimmune uveitis: Tcell heterogeneity, migration, and steroid response. European Journal of Immunology. 50 (12), 1941-1951 (2020).

8. $\mathrm{Xu}, \mathrm{H}$. et al. A clinical grading system for retinal inflammation in the chronic model of experimental autoimmune uveoretinitis using digital fundus images. Experimental Eye Research. 87, 319-326 (2008).

9. Copland, D. A. et al. The clinical time-course of experimental autoimmune uveoretinitis using topical endoscopic fundal imaging with histologic and cellular infiltrate correlation. Investigative Ophthalmology \& Visual Science. 49, 5458-5465 (2008).

10. Dick, A. D. Doyne lecture 2016: intraocular health and the many faces of inflammation. Eye (London). 31, 87-96 (2017).

11. Agarwal, R. K., Silver, P. B., \& Caspi, R. R. Rodent models of experimental autoimmune uveitis. Methods in Molecular Biology. 900, 443-469 (2012).

12. Caspi, R. R. A look at autoimmunity and inflammation in the eye. Journal of Clinical Investigation. 120, 3073-3083 (2010).

13. Caspi, R. R. et al. Mouse models of experimental autoimmune uveitis. Ophthalmic Research. 40, 169-174 (2008).
14. Shao, H. et al. Severe chronic experimental autoimmune uveitis (EAU) of the C57BL/6 mouse induced by adoptive transfer of IRBP1-20-specific T cells. Experimental Eye Research. 82, 323-331 (2006).

15. Horai, R. et al. Microbiota-Dependent Activation of an Autoreactive T Cell Receptor Provokes Autoimmunity in an Immunologically Privileged Site. Immunity. 43, 343-353 (2015).

16. Chen, J. et al. Comparative analysis of induced vs. spontaneous models of autoimmune uveitis targeting the interphotoreceptor retinoid binding protein. PLoS One. 8, e72161 (2013).

17. Chen, J., Qian, H., Horai, R., Chan, C. C., \& Caspi, R. R. Use of optical coherence tomography and electroretinography to evaluate retinal pathology in a mouse model of autoimmune uveitis. PLoS One. 8, e63904 (2013).

18. Harry, R. et al. Suppression of autoimmune retinal disease by lovastatin does not require Th2 cytokine induction. Journal of Immunology. 174, 2327-2335 (2005). 